Alberta-Thy-16-94

\title{
Mass Inflation in a Rotating Charged Black Hole
}

\author{
Alfio Bonanno \\ Canadian Institute for Advanced Research Cosmology Program, \\ Theoretical Physics Institute, University of Alberta, \\ Edmonton, Alberta, Canada T6G 2J1 \\ and \\ Institute of Astronomy, University of Catania \\ Viale Andrea Doria 6, 95125 Catania, Italy
}

PACS numbers: 97.60Lf,04.70.-s,04.20.Dw

\begin{abstract}
The structure of the Cauchy horizon of a charged rotating black hole is analyzed under the combined effect of an ingoing and outgoing flux of gravitational waves. In particular, by means of an axisymmetric realization of the Ori model, the growth of the mass parameter near the Cauchy horizon is studied in the slow rotation approximation. It is shown that the mass-parameter inflates, while the angular momentum per unit mass deflates, but initial deviations from spherical symmetry survive.
\end{abstract}


Although it is now generally accepted that gravitational collapse results in the formation of a black hole, the ultimate fate of the collapsing object within the black hole is an open question. The presence of an inner horizon, - the Cauchy horizon $(\mathrm{CH})$ - a lightlike surface behind which the predictability of the field equations breaks down, turns out to be a formidable obstacle to constructing an unambiguous picture of the complete analytical extension of the geometry. This fundamental issue is encoded in the peculiar character of the $\mathrm{CH}$.

As noted first by Penrose [1], ingoing pencils of radiation experience a diverging blueshift as they approach the generators of the $\mathrm{CH}$. This kind of nonscalar singularity, known as a "whimper" [2], is unstable to perturbations. Then a stronger, scalar singularity can develop when the backreaction of the fields on the metric is taken into account. If the additional effect of an outgoing flux is considered, spherical models of the crossflow region [3] show that the effective mass parameter $m(u, v)$ exponentially inflates at late advanced times, as the $\mathrm{CH}$ is approached. In particular the Weyl curvature invariant $\Psi_{2}$ diverges, indicating that a scalar singularity occurs. But this divergence is still "mild", since the mass function is an integrable function of the Kruskalized advanced coordinate, and in suitable coordinates the metric coefficients stay finite at the $\mathrm{CH}$.

How general are these models in describing the evolution of the interior at late advanced times, if the restriction of spherical symmetry is removed? General arguments based on the constancy of the surface gravity over a stationary horizon [3] indicate that the growth of the mass parameter should appear uniform on small angular scales. One suspects in particular that in a generic axisymmetric collapse the "effective Kerr parameter" (the angular momentum per unit mass $a=J / m$ ) becomes negligible if the total angular momentum of the inflalling radiation is bounded during the collape [5]. The asymptotic structure of the spacetime close to the $\mathrm{CH}$ should look like an axially symmetric geometry with an enormously inflated mass term. Examples of axisymmetric mass-inflation solutions have been discussed in $2+1$ models [6], however the resulting Kretschmann invariant is found to be finite at the singularity unlike the spherically symmetric model in $3+1$ dimensions. A more realistic analysis of the instability of the $\mathrm{CH}$ for a class of KerrNewman spacetime has been proposed in [7], and in the framework of the $2+2$ approach in [8]. In this latter analysis the resulting asymptotic configuration seems not to be that of a Petrov type D spacetime.

A possible insight into the question lies in the nature of the mass-inflation phenomenon. The out- going flux is a catalyzer which causes the generators to contract without a direct interaction with the infinitely blueshifted infalling lightlike contribution. The rate of contraction is fully determined by Price's power law damping of the radiative tail $\sim 1 / v^{(p-1)}$, $p \geq 11$. Hence one can argue that deviations from the purely spherical geometry of the $\mathrm{CH}$ should be reflected in deviation from spherical symmetry in the mass-inflated sector, since the contraction will not be uniform in a non-spherical model. The leading contribution to the mass function should then be dominated by a very large mass term with a small angular dependence. We shall here present an explicit massinflation solution in the case of a rotating charged hole that exhibits this behavior. Our model, although approximate, should capture the qualitative features of the geometry of the $\mathrm{CH}$ in a non-stationary, rotating black hole.

The crossflow region, near the $\mathrm{CH}$, is described by an outgoing lightlike shell - simulating the outgoing flux - embedded in a continuous flow of infalling gravitational waves, see Fig.1 . This axisymmetric realization of the Ori [9] model is derived in the slow rotation approximation when $a=\frac{J}{m}$ is small compared to the radius of the $\mathrm{CH} r_{0}$

$$
\epsilon \equiv \frac{a^{2}}{r_{0}^{2}} \ll 1
$$

In particular, as background geometry we consider the non-stationary Vaidya type generalization of the Kerr metric discussed in [10]. We extend that model to the charged case in order to retain a finite (nonzero) radius for the inner horizon even when $a$ is small. In the $\{r, \vartheta, \varphi, v\}$ Eddington-Kerr coordinate system the metric reads

$$
\begin{aligned}
& d s^{2}=-\left(1-\frac{2 m(v) r-e^{2}}{\Sigma}\right) d v^{2}+2 d r d v \\
& +\Sigma d \vartheta^{2}-2 a \sin ^{2} \vartheta d \varphi d r+\mathcal{R}^{2} \sin ^{2} \vartheta d \varphi^{2} \\
& -\frac{2 a\left(2 m(v) r-e^{2}\right) \sin ^{2} \vartheta}{\Sigma} d v d \varphi
\end{aligned}
$$

where

$$
\begin{aligned}
& \mathcal{R}^{2}=\frac{\left(r^{2}+a^{2}\right)^{2}-\Delta a^{2} \sin ^{2} \vartheta}{\Sigma}, \\
& \Sigma=r^{2}+a^{2} \cos ^{2} \vartheta \\
& \Delta=r^{2}+a^{2}+e^{2}-2 m(v) r
\end{aligned}
$$

As in the spherical Vaidya model, the mass parameter $m(v)$ is a function of the advanced coordinate $v$. Its 
functional dependence near the $\mathrm{CH}$, located at $v=$ $+\infty$, is assumed to be of the form

$$
m=m_{0}+\delta m(v), \quad \delta m(v)=-\frac{A}{v^{p-1}}
$$

to reproduce the power law decay of the gravitational waves. Although in a realistic setting the mass function has probably an angular dependence as well, the assumption (4) is physically reasonable since we work in the slow rotation approximation, and (4) is the only relevant contribution. In the following analysis it is convenient to introduce the null complex tetrad $\left\{l_{\mu}, n_{\nu}, m_{\mu}, \bar{m}_{\mu}\right\}$, with $-l_{\mu} n^{\mu}=m_{\mu} \bar{m}^{\mu}=1$ and $l_{\mu}$ is the repeated principal null direction associated with the infalling field $-r$ decreases with time along $v=$ const. -

$$
\begin{aligned}
& l_{\mu}=-\partial_{\mu} v+a \sin ^{2} \vartheta \partial_{\mu} \varphi \\
& n_{\mu}=-\frac{1}{\Sigma}\left(\frac{\Delta}{2} \partial_{\mu} v-\Sigma \partial_{\mu} r-a \sin ^{2} \vartheta \frac{\Delta}{2} \partial_{\mu} \varphi\right) \\
& m_{\mu}=-\frac{\bar{\rho}}{\sqrt{2}}\left(i a \sin \vartheta \partial_{\mu} v+\Sigma \partial_{\mu} \vartheta\right. \\
& \left.-i\left(r^{2}+a^{2}\right) \sin \vartheta \partial_{\mu} \varphi\right)
\end{aligned}
$$

where $\rho=-(r-i a \cos \vartheta)^{-1}$. The total energy momentum tensor can be expressed as

$$
\begin{aligned}
& T_{\mu \nu}=2 \phi_{22} l_{\mu} l_{\nu}-4 \phi_{12} l_{(\mu} \bar{m}_{\nu)}-4 \bar{\phi}_{12} l_{(\mu} m_{\nu)} \\
& +4 \phi_{11}\left(l_{(\mu} n_{\nu)}+m_{(\mu} \bar{m}_{\nu)}\right)
\end{aligned}
$$

where $\phi_{22}, \phi_{12}, \phi_{11}$ are the only non-vanishing tetrad components of the trace free part of the Ricci tensor $S_{\mu \nu}=R_{\mu \nu}-1 / 4 g_{\mu \nu} R,\left(=R_{\mu \nu}\right.$ in our case),

$$
\begin{aligned}
& \phi_{22}=r\left(2 \dot{m}(v)-a^{2} \sin ^{2} \vartheta \ddot{m}(v)\right) / 4 \Sigma^{2} \\
& \phi_{11}=e^{2} / 2 \Sigma^{2} \\
& \phi_{12}=-i a \sin \vartheta \dot{m}(v) \rho / 2 \sqrt{2} \Sigma
\end{aligned}
$$

The radiation field consists of a pure null part and a residual term. In particular $\phi_{11}$ represents the contribution of a static source field generated by a charge of strength $e$. Unlike the stationary case, there is only one repeated principal null direction: the spacetime is algebraically special of Petrov type II and the only non-zero Weyl invariants are

$$
\begin{aligned}
& \Psi_{2}=-m(v) \rho^{3}-e^{2} \rho \bar{\rho}^{3} \\
& \Psi_{3}=\frac{-i \dot{m}(v) \rho a \sin \vartheta}{2 \sqrt{2} \Sigma}-\frac{i \dot{m}(v) \rho^{2} r a \sin \vartheta}{\sqrt{2} \Sigma} \\
& \Psi_{4}=\frac{\ddot{m}(v) \rho^{2} r a^{2} \sin ^{2} \vartheta}{\sqrt{2} \Sigma}+\frac{\dot{m}(v) \rho^{3} r a^{2} \sin ^{2} \vartheta}{\sqrt{2} \Sigma}
\end{aligned}
$$

Let us now consider the equation of motion $r=$ $r(v, \vartheta)$ of an axisymmetric generic outgoing null hypersurface. Close to $\mathrm{CH}$, where the functional dependence of the mass function on the advanced coordinate is given by (4), it reads

$$
\begin{aligned}
& -\frac{2 \delta m(v) r}{r^{2}+a^{2}}-2 \kappa_{0}\left(r-r_{0}\right)-2 \partial_{v} r \\
& +\frac{a^{2} \sin ^{2} \vartheta}{r^{2}+a^{2}}\left(\partial_{v} r\right)^{2}+\frac{\left(\partial_{\vartheta} r\right)^{2}}{r^{2}+a^{2}}=0
\end{aligned}
$$

where $\kappa_{0}$ is the surface of gravity of the inner horizon located at $r=r_{0}$. To first order in the effective rotation parameter $\epsilon$, the solution of has to be of the form

$$
r-r_{0}=f(v)+\epsilon g(v, \vartheta)+O\left(\epsilon^{2}\right)
$$

and (9) is equivalent to the following system of partial differential equations for the zeroth and first order terms respectively

$$
\begin{aligned}
& \kappa_{0} f+f_{v}=-\frac{\delta m(v)}{r} \\
& \kappa_{0} g+g_{v}-\frac{1}{2} \sin ^{2} \vartheta f_{v}^{2}=\frac{\delta m(v)}{r}
\end{aligned}
$$

The boundary conditions

$$
\lim _{v \rightarrow \infty} f(v)=g(v, \vartheta)=0
$$

determine the following asymptotic form for the solution as $v \rightarrow \infty$ :

$$
r-r_{0}=f(v)\left(1-\epsilon\left(\frac{\sin ^{2} \vartheta(p-1)}{2 \kappa_{0} v}+O\left(\epsilon / v^{2}\right)\right)\right)
$$

where

$$
f(v)=\frac{A}{\kappa_{0} r_{0}} v^{-(p-1)}\left(1+\frac{p-1}{\kappa_{0} v}+\ldots\right)
$$

This shows that the angular dependence is suppressed by a factor $1 / v$ to first order in $\epsilon$ as we approach the $\mathrm{CH}$.

Now we consider this hypersurface to be the locus of a lightlike shell embedded in this background. The spacetime is then divided in two regions $\mathcal{M}^{+}$, and $\mathcal{M}^{-}$, see Fig.1, separated by the outgoing shell $\mathcal{S}$ whose equation of motion is of the form (13) near the $\mathrm{CH}$. We assume that the "past" side of the shell is described by the radiating Kerr-Newman metric (2). In general nothing can be said about the future side of $\mathcal{S}$. However, it is reasonable to think that in the slow rotation regime the production of gravitational waves can be neglected and the structure of the resulting "glued" manifold is still Kerr-like at least close 
to CH. Thus as "trial" metric we assume that the spacetime in the future sector of the shell can be represented with a line element of the type (2) where the coefficients functions $r_{+}, a_{+}, m_{+}$depend on the coordinates in $\mathcal{M}^{-}$.

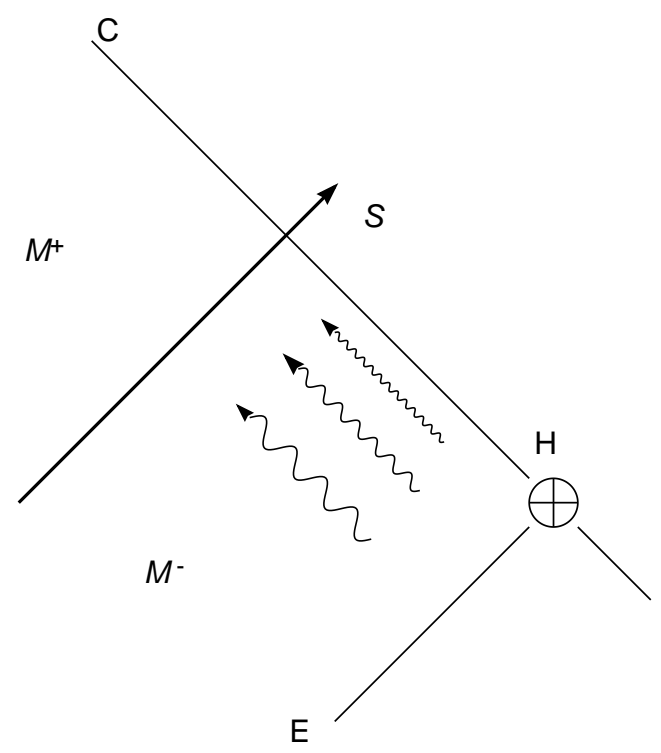

Fig. 1. A spacetime diagram of the equatorial plane of the axisymmetric black hole interior showing the ingoing flow of gravitational waves being crossed by an outgoing lightlike shell infalling in the Cauchy horizon.

In particular let $\left\{v_{ \pm}, r_{ \pm}, \vartheta_{ \pm}\right\}$be the local coordinates of $\mathcal{S}$ in $\mathcal{M}^{ \pm}, a_{ \pm}$the value of the angular momentum per unit mass, and $m_{ \pm}$the mass functions in both the sides of the shell. The stress-energy tensor in $\mathcal{M}^{+}$contains the contribution in (6) and residuals terms arising from the fact that $a_{+}$is not stricly constant. Those terms can be shown to be much smaller than the leading, optical geometric contribution in (6) close to $\mathrm{CH}$. We shall explicitly check the validity of this approximation at the end of the computation.

In this model the presence of the outgoing lightlike shell simply serves to start the contraction of the generators of the $\mathrm{CH}$. Thus we consider a pressureless shell so that the soldering of the two geometries is affinely conciliable [11]. We remark that since the analysis in [12, 13] shows that for spherical symmetry the $\mathrm{CH}$ survives the focusing effect of the outgoing flux, it is reasonable to think that this would be the case even if the hole is slowly rotating. In order to isolate the divergent contribution in the mass function in $\mathcal{M}^{+}$we define $m_{+}=\bar{m}+M\left(v_{+}\right)$where, by definition $2 \bar{m} \bar{r}=\bar{r}^{2}+\bar{a}^{2}+e^{2}$ and $\bar{r}, \bar{a}$ are the values of $r_{+}$and $a_{+}$at the $\mathrm{CH}$. As before, the solution of
(9) in $\mathcal{M}^{+}$is of the form

$$
r_{+}-r_{0+}=f_{+}\left(v_{+}\right)+\frac{a_{+}^{2}}{r^{2}} g_{+}\left(v_{+}, \vartheta_{+}\right)
$$

Therefore equation (15) decouples as a system of the type (11). In particular if $M\left(v_{+}\right) \gg r_{0}$ near the $\mathrm{CH}$, at the leading order in the mass term it reduces to

$$
\frac{\partial f_{+}}{\partial v_{+}} \simeq-\frac{M_{+}}{r_{0+}}, \quad \frac{\partial g_{+}}{\partial v_{+}} \simeq \sin ^{2} \vartheta_{+} \frac{M^{2}\left(v_{+}\right)}{r_{0+}^{2}}
$$

The only geometric condition that has to be satisfied along $\mathcal{S}$, the common boundary of the two spacetimes, is that the two intrinsic degenerate metrics coincide. This implies that the area $\mathcal{A}$ of the two intrinsic metrics has to be continuous across the shell,

$$
[\mathcal{A}]=0
$$

where $[\mathcal{A}]=\mathcal{A}_{+}-\mathcal{A}_{-}$. In a perturbative expansion in $a_{ \pm}$, this condition decouples into two distinct continuity requirements for the zeroth and first order terms. The spherically symmetric contribution simply states the continuity of the $r$ coordinate across $\mathcal{S}$, not a priori guaranteed from (17). Thus $r_{+}=r_{-}=r$ and we set $r_{0+}=r_{0-}=r_{0}$ at the $\mathrm{CH}$. We use $r$ as a parameter (necessarily affine for a pressureless shell) along the generators of $\mathcal{S}$ By using eq. (13) in $\mathcal{M}^{-}$one finds that along the shell the area of any $v_{-}=$const. hypersurface reads

$$
\mathcal{A}_{-}=4 \pi r_{0}^{2}(1+\epsilon)+O\left(1 / v^{p}\right), \quad v_{-} \rightarrow+\infty
$$

where only linear term in $\epsilon$ have been retained in the degenerate metric. Similarly in $\mathcal{M}^{+}$, close to the $\mathrm{CH}$ and up to linear terms to $a_{+}^{2}$, one has

$$
\mathcal{A}_{+}=4 \pi\left(r_{0}^{2}+a_{+}^{2}\left(1+\frac{2 M\left(v_{+}\right)}{3 r_{0}}\right)\right)
$$

In particular, if $M\left(v_{+}\right) / r_{0} \gg 1$, equation (17) reads

$$
a_{+}^{2} M_{+} \simeq 3 \epsilon r_{0}^{3} / 2, \quad v_{-} \rightarrow+\infty
$$

This latter equation contains the essential physics. As observed in the beginning, it explicitly shows that the effective Kerr parameter $a_{+}$becomes increasingly small as the mass function grows. We stress, however, that the asymptotic geometry close to the $\mathrm{CH}$ is not spherically symmetric because the $a_{+}^{2} M_{+}$and $a_{+} M_{+}$ terms in the metric are not negligible. The dependence of $v_{+}$and $\vartheta_{+}$on the advanced coordinates in the past sector of the shell can be determined from the expression for the null generators on both sides of $\mathcal{S}$ (the dependence of $\varphi_{+}$on $\varphi_{-}$is trivial since these coordinate define the same Killing vector). From the 
chain rule and the continuity of the $r$ function one has

$$
\begin{aligned}
& \partial_{v} f_{-}=\partial_{v} f_{+}\left[\frac{\partial v_{+}}{\partial v_{-}}\right]^{(0)} \\
& \epsilon \partial_{v} g_{-}=\partial_{v} f_{+}\left[\frac{\partial v_{+}}{\partial v_{-}}\right]^{(1)}+\frac{a_{+}^{2}}{r_{0}^{2}} \partial_{v} g_{+}\left[\frac{\partial v_{+}}{\partial v_{-}}\right]^{(0)} \\
& \epsilon \partial_{\vartheta} g_{-}=\partial_{v} f_{+}\left[\frac{\partial v_{+}}{\partial \vartheta_{-}}\right]^{(1)}+\frac{a_{+}^{2}}{r_{0}^{2}} \partial_{\vartheta} g_{+}
\end{aligned}
$$

where only first order terms in the jacobian determinant have been retained. To relate the dynamics of the two spacetime one has to add the condition for matching of normal stresses across the shell

$$
\left[T^{\mu \nu} s_{\mu} s_{\nu}\right]=0
$$

where $s_{\mu}$ are generators of $\mathcal{S}$. Equation $(22)$ is a second order ordinary differential equation for $M_{+}$. The dependence of $\left\{v_{+}, \vartheta_{+}\right\}$on the local coordinates in $\mathcal{M}_{-}$is implicitly defined from (21), and from the equations of motion of $\mathcal{S}$ in the two spacetimes. The explicit solution of (22) is not available. However in the slow rotation approximation any scalar product between the generators $s^{\mu}$ and the tetrad vector must be written as a sum of a function of only the advanced coordinated plus $\epsilon$ times a function of both advanced and angular coordinates, and (22) reduces to

$$
\left[\phi_{22} l_{\mu} l_{\nu} s^{\mu} s^{\nu}\right]=0
$$

where the scalar products have to be calculated from the spherically symmetric contribution. Therefore it is important to stress that in this approximation only the optical, physically meaningful, part of the energy momentum tensor is relevant. This equation contains the coupling between the angular momentum and mass function, through the $a_{+} \ddot{M}_{+}$term. By using (21) in (23) and by expressing $a_{+}$with the help of eq. (20), after some simplifications one explicitly finds

$$
\begin{aligned}
& {\left[\frac{d^{2} M_{+}}{d v_{-}^{2}}+\frac{1}{M_{+}}\left(\frac{d M_{+}}{d v_{-}}\right)^{2}\right] \frac{3 \epsilon r_{0} \sin ^{2} \vartheta}{4 \partial_{v} f_{-}}-\frac{d M_{+}}{d v_{-}}} \\
& =-\kappa_{0}\left(1-p \frac{4+\kappa_{0} r_{0} 3 \epsilon \sin ^{2} \vartheta}{4 \kappa_{0} v_{-}}\right) M_{+}
\end{aligned}
$$

note that $\vartheta_{-}=\vartheta_{+}$in this approximation. Hence we write

$$
M_{+}=m_{+}+\delta m_{+}
$$

and we have from (24)

$$
m_{+}=\frac{1}{v_{-}^{p}} e^{\kappa_{0} v_{-}}, \quad v_{-} \rightarrow+\infty .
$$

This has the Israel-Poisson behavior, and

$$
\delta m_{+} \sim c \epsilon \sin ^{2} \vartheta e^{\kappa_{0} v_{-}}
$$

where $c$ is a constant. This shows the effective mass parameter exponentially inflates with a residual angular dependence

$$
M_{+} \sim e^{\kappa_{0} v_{-}}\left(1+c \epsilon \sin ^{2} \vartheta\right)
$$

to first order in $\epsilon$, that does not effect the exponentially divergent prefactor [3]. From the equations (21) one finds

$$
\begin{aligned}
& {\left[\frac{\partial v_{+}}{\partial v_{-}}\right]^{(0)} \sim e^{-\kappa_{0} v_{-}}, \quad\left[\frac{\partial v_{+}}{\partial v_{-}}\right]^{(1)} \sim \epsilon \sin ^{2} \vartheta_{-} e^{-\kappa_{0} v_{-}}} \\
& {\left[\frac{\partial v_{+}}{\partial \vartheta_{-}}\right]^{(1)} \sim \epsilon \kappa_{0}^{-1} \sin 2 \vartheta e^{-\kappa_{0} v_{-}}}
\end{aligned}
$$

We see that the radial coordinate tends to a finite limit behind the shell. Indeed, from eq. (16), we have

$$
\begin{aligned}
& f\left(v_{+}\right) \sim \frac{1}{\ln ^{p}\left|v_{+}\right|}, \quad v_{+} \rightarrow 0 \\
& a_{+}^{2} g\left(v_{+}, \vartheta_{+}\right) \sim \frac{r_{0}^{2} \sin ^{2} \vartheta_{+}}{\ln ^{p}\left|v_{+}\right|}, \quad v_{+} \rightarrow 0
\end{aligned}
$$

thus,

$$
\lim _{v_{+} \rightarrow 0} r\left(v_{+}\right)=r_{0}
$$

The geometry in the mass-inflated sector is asymptotically dominated by the large mass term, and the metric, to a good approximation, explicitly reads

$$
\begin{aligned}
& d s_{+}^{2} \approx \frac{2 m_{+}}{r}\left(1+c \epsilon \sin ^{2} \vartheta\right) d v_{+}^{2}+2 d r d v_{+}+r^{2} d \vartheta^{2} \\
& +r^{2}\left(1+3 \epsilon \sin ^{2} \vartheta\right) \sin ^{2} \vartheta d \varphi^{2} \\
& -4 \sqrt{3 \epsilon m_{+} r / 2} \sin ^{2} \vartheta d v_{+} d \varphi
\end{aligned}
$$

The following "mild" twist of the $\varphi$ coordinate (since $m_{+}$is an integrable function of the advanced coordinate $v_{+}$)

$$
d \varphi=d \Phi+\frac{2 \sqrt{3 \epsilon m_{+} r_{0} / 2}}{r_{0}^{2}} d v_{+}
$$

brings the metric in the final form

$$
\begin{aligned}
& d s_{+}^{2} \approx \frac{2 m_{+}}{r}\left(1+c \epsilon \sin ^{2} \vartheta\right) d v_{+}^{2}+2 d r d v_{+} \\
& +r^{2} d \vartheta^{2}+r^{2}\left(1+3 \epsilon \sin ^{2} \vartheta\right) \sin ^{2} \vartheta d \Phi^{2}
\end{aligned}
$$

to linear terms in $\epsilon$. This result is interesting, we believe, because it explicitly shows that deviations 
from spherical symmetry at the Cauchy horizon are reflected in the mass-inflated sector, in accordance with the remarks at the beginning. This phenomenon should be characteristic of the dynamics of a spacetime with a non-spherical CH. It is important to check the consistency of the approximations that have been done. In fact had we started with the line element (34), with $m_{+}=v_{+}^{-1} \ln ^{-p}\left|v_{+}\right|$we would find

$$
\mathcal{A}_{+}=4 \pi r_{0}^{2}(1+\epsilon)=\mathcal{A}_{-}+O\left(1 / v^{p}\right)
$$

for the continuity of the intrinsic area across the shell. Also, it is straightforward to verify that the stressenergy tensor computed from (34) close to $\mathrm{CH}$ satisfies the matching condition for the normal stresses, equation (22)

$$
\left[T^{\mu \nu} s_{\mu} s_{\nu}\right]=O\left(\epsilon^{2}\right) .
$$

The Komar invariant quantity associated with the rotational Killing vecor field $\xi^{\mu}$ is not conserved since matter is flowing into the system. It is indeed divergent in our model, but with a much slower rate

$$
\frac{1}{8 \pi} \oint \xi^{\mu ; \nu} d \sigma_{\mu \nu} \sim \sqrt{m_{+}}
$$

where the integral is taken over the two dimensional boundary of any $v_{-}=$const. hypersurface, with $v_{-} \rightarrow+\infty$. It is however hard to judge, from the analysis here presented, whether one can expect this latter result to occur in a more general framework than that of our model. In particular at the present we do not see any deeper physical argument to explain it.

As in the sperically symmetric models, at the $\mathrm{CH}$ a strong, scalar singularity develops, whose character can be read off from the Weyl curvature invariants in (8). One finds that to the future of the shell, they are all divergent:

$$
\begin{aligned}
\Psi_{2} & \sim \frac{1}{v_{+} \ln ^{p}\left|v_{+}\right|}, \quad \Psi_{3} \sim \frac{1}{v_{+} \sqrt{\left|v_{+}\right|} \ln ^{p / 2}\left|v_{+}\right|} \\
\Psi_{4} & \sim \frac{1}{v_{+}^{2} \sqrt{\left|v_{+}\right|} \ln ^{p / 2}\left|v_{+}\right|}
\end{aligned}
$$

Although $\Psi_{3}$ and $\Psi_{4}$ are tetrad-dependent, the divergence of the boost-invariant quantity $\Psi_{2}$ has the same "mild" trait as in the spherically symmetric case.

Finally, we remark that these results have been derived in the slow rotation approximation and it would be interesting to see how this scenario would evolve in the more general case of arbitrary spin.

The author would like to thank Werner Israel for his constant advice and many enlightening discus- sions, as well Valery Frolov, Sharon Morsink, Charles Torre, David Hobill, Roberto Balbinot and Eric Poisson, for many useful and important comments. This work has been supported by the Italian Minister for the University and Scientific Research,and by the Institute of Astronomy of the University of Catania.

\section{References}

[1] R.Penrose in Battelle Rencontres, ed. C.M.DeWitt and J.A.Wheeler, W.A.Benjamin, New York, 1968, p.222

[2] G.F.R. Ellis and A.R. King, Commun. Math. Phys. 38, 119 (1974). A.R. King, Phys. Rev. D 11, 763 (1975).

[3] E. Poisson and W. Israel, Phys. Rev. D41, 1796, (1990).

[4] P.R.Brady and J.D. Smith, "Black hole singularities: a numerical approach" Univ. of NewcastleUpon-Tyne, to appear in Physical Review Letters.

[5] C.Barrabes, W. Israel, E. Poisson, Class. Quantum Grav., 7, L273 (1990).

[6] J.S.Chan,K.C.Chan,R.B.Mann, preprint WATPHYS TH-94/06

[7] D.A.Konkowski, T.M.Helliwell, Phys.Rev.D, (1994) 50, 841

[8] P.R.Brady and C.M.Chambers, preprint NCL94TP13

[9] A.Ori, Phys.Rev.Letters, 67, 1991, 789

[10] M.Carmeli and M.Kaye, Annals of Physics, 103, 97, (1977)

[11] W.Israel and C.Barrabés, Physical Review D, 43, 1129, (1991)

[12] A.Bonanno, S.Droz, W.Israel and S.M.Morsink, Phys.Rev.D, (1994) 50, 7372

[13] A.Bonanno, S.Droz, W.Israel and S.M.Morsink, Proc.R.Soc.Lond. A, to appear 